\title{
ARTÍCULOS
}

\section{Modelos fustales para renovales de roble, raulí y coigüe en Chile}

\author{
Taper models for roble, raulí and coigüe second growth forests in Chile \\ Salvador A Gezan ${ }^{a *}$, Paulo C Moreno $\mathbf{M}^{\mathrm{b}}$, Alicia Ortegac

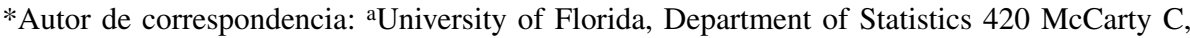 \\ PoBox 110339, Gainesville, FL 32611, sgezan@ufl.edu \\ bInstituto Forestal, Coyhaique, Chile. \\ ${ }^{c}$ Universidad Austral de Chile, Facultad de Ciencias Forestales, Valdivia, Chile.
}

\begin{abstract}
SUMMARY
A total of ten individual tree taper models were fitted to felled raulí (Nothofagus alpina), roble (N. obliqua) and coigüe (N. dombeyi) trees, corresponding to total of 558 individuals (251, 180 and 127, respectively). They were selected from 108 sample plots of pure or mixed roble-raulí-coigüe second growth forests located between $36^{\circ}$ and $42^{\circ} \mathrm{S}$ latitude in Chile. The taper models and a volume model were fitted to 12 fitting groups; these were defined according to the combinations of growth zone and specie together with three general groups representing each species. In all groups, the best taper models allowed to describe the profile with error values smaller than $15 \%$ in the estimation of the without bark section diameter. In all cases, the precision and bias in the volume predictions using taper models were similar to the individual volume model. At a general level, small differences among the best models were found with better results for Bruce and Kozak models where the latter showed slightly better performance.
\end{abstract}

Key words: Nothofagus, tapering, volume, growth zones.

\section{RESUMEN}

Un total de diez modelos fustales de árbol individual fueron ajustados para árboles volteados de raulí (Nothofagus alpina), roble (N. obliqua) y coigüe ( $N$. dombeyi), correspondiendo a un total de 558 individuos $(251,180$ y 127, respectivamente) provenientes de 108 parcelas de muestreo en rodales puros o mixtos de renovales de roble-raulí-coigüe localizados entre los $36^{\circ}$ y $42^{\circ} \mathrm{S}$ en Chile. Los modelos fustales y un modelo de volumen fueron ajustados para 12 grupos, los que fueron definidos con base en la combinación de zona de crecimiento y especie, además de tres grupos generales que representan a cada especie. En todos los grupos, los mejores modelos fustales permitieron describir el perfil con valores de error menores al 15\% en la estimación del diámetro de sección sin corteza. En todos los casos, la precisión y sesgo de las predicciones de volumen utilizando modelos fustales fueron similares al modelo de volumen individual. A nivel general, se encontraron leves diferencias entre los mejores modelos, destacando el de Bruce y Kozak con resultados ligeramente mejores para este último.

Palabras clave: Nothofagus, ahusamiento, volumen, zonas de crecimiento.

\section{INTRODUCCIÓN}

Los modelos fustales o funciones de ahusamiento son comúnmente utilizados en inventarios forestales para estimar volúmenes de trozas o productos. Ellos corresponden a funciones matemáticas que describen el diámetro o perfil del árbol a lo largo del fuste y dependen de variables tales como el diámetro a la altura del pecho y la altura total. Entre otras cosas, los modelos fustales permiten estimar diámetros a cualquier altura del fuste, altura de un diámetro límite dado y volúmenes de productos definidos entre dos puntos del fuste (Newnham 1992, Prodan et al. 1997). Además, constituyen un componente importante en los modelos de crecimiento y rendimiento y en los simuladores de trozado. Debido a la dificultad en describir la forma de un árbol en forma precisa, diversas clases de modelos han sido propuestas en la literatura, tales como los polinómicos (Bruce et al. 1968), trigonométricos (Thomas y Parresol 1991), de forma o exponente variable (Kozak 1988), segmentados (Max y Burkhart 1976) y compatibles con funciones de volumen individual (Goulding y Murray 1976).

Los renovales de roble (Nothofagus obliqua (Mirb.) Oerst.), raulí (Nothofagus alpina (Poepp et Endl.) Oerst.) y coigüe (Nothofagus dombeyi (Mirb.) Oerst.) representan un recurso de alto valor económico y ecológico. En Chile, los bosques del tipo forestal roble-raulí-coigüe, que se localizan desde la Región del Maule a la de Los Lagos, cubren una superficie cercana a los 1,2 millones de hectáreas (CONAF et al. 1999). La aplicación de un manejo sustentable de este recurso forestal requiere de un conocimiento acabado de las existencias a nivel de rodal o árbol individual. En este sentido, un elemento importante es la disponibilidad 
de modelos de volumen o ahusamiento para determinar existencias. Para coigüe no existen modelos publicados al presente y Kahler (1993) reportó el ajuste de diversos modelos fustales para renovales de roble y raulí. Debido a que estos modelos fueron obtenidos sobre la base de muestras provenientes de áreas geográficas relativamente reducidas, su uso generalizado, particularmente para condiciones ambientales diferentes, requiere de un estudio de validación con muestras independientes.

Este estudio se enmarca dentro de un proyecto de simulación de crecimiento y rendimiento para renovales de roble, raulí y coigüe (Ortega y Gezan 1998) en donde los modelos de ahusamiento constituyen un componente importante para determinar existencias volumétricas. El objetivo de este estudio es obtener modelos fustales a partir del volteo de árboles seleccionados sobre la base de un muestreo extensivo de renovales que abarcan casi toda su distribución en Chile. De este modo, el principal aporte de este trabajo es contribuir con antecedentes basados en lo que es, a la fecha, la base muestral más amplia de este recurso forestal.

\section{MÉTODOS}

Los datos utilizados en este estudio corresponden a árboles volteados a los cuales se les midió su altura total (h), diámetro a la altura del pecho (d) y diámetros de sección sin corteza $\left(d_{l}\right)$ a distintas alturas en el fuste $\left(h_{l}\right)$. Se recopiló información de tres estudios diferentes considerando parcelas localizadas entre los $36^{\circ}$ y $42^{\circ} \mathrm{S}$ en Chile. La primera fuente correspondió a 24 parcelas de $1.000 \mathrm{~m}^{2}$ (99 árboles) provenientes de un estudio de crecimiento de renovales de roble y raulí (Donoso et al. 1993). Estas parcelas fueron establecidas en localidades sin evidencia de alteración antrópica, en donde tres individuos del dosel dominante fueron volteados, a los cuales se les extrajeron rodelas a $0,3 \mathrm{~m}, 1,3 \mathrm{~m}, 2 \mathrm{~m}$, y cada 2 metros hasta la altura total. La segunda fuente constó de 28 parcelas de superficies entre 500 y $1.000 \mathrm{~m}^{2}$ (272 árboles) establecidas en renovales de roble, raulí, coigüe y tepa (Grosse y Cubillos 1991). Los árboles seleccionados correspondieron a aquellos marcados para ralear bajo el criterio del árbol futuro. A cada uno de ellos se le extrajeron rodelas a 0,3 $\mathrm{m}, 1,3 \mathrm{~m}, 2,3 \mathrm{~m}$ y cada $2 \mathrm{~m}$ hasta un diámetro límite de $5 \mathrm{~cm}$. La última fuente correspondió a 56 parcelas temporales de $250 \mathrm{~m}^{2}$ (167 árboles) provenientes del proyecto FONDEF D97I1065 (Ortega y Gezan 1998). Estas fueron establecidas considerando un muestreo proporcional a la superficie y distribución geográfica del recurso de acuerdo a las estadísticas reportadas por CONAF et al. (1999) para este recurso. Los rodales muestreados presentaron nulos o bajos niveles de alteración y en cada uno se voltearon cuatro árboles (dos dominantes, uno intermedio y uno suprimido) y se extrajeron rodelas cada $1 \mathrm{~m}$ desde los 0,3 $\mathrm{m}$ hasta un diámetro límite de $5 \mathrm{~cm}$.
De este modo, la base de datos contó con un total de 558 árboles volteados (251 de raulí, 180 de roble y 127 de coiguie) distribuidos en 108 parcelas, correspondiendo a 6.420 rodelas (cuadro 1). Sólo se seleccionaron árboles que presentaron una razón $h / d$ entre 0,7 y 1,7 a fin de eliminar aquellos con ápices quebrados o creciendo en condiciones adversas. Un total de 12 grupos de ajuste fue considerado para la obtención de modelos. Estos fueron definidos de acuerdo a la combinación de especie y zona de crecimiento con una cantidad mínima de 20 árboles. Adicionalmente se consideraron tres grupos generales correspondientes a cada especie (cuadro 1). Las zonas de crecimiento fueron definidas usando criterios ambientales tales como: límites climáticos y altitudinales, cortes geomorfológicos, material de origen del suelo, condiciones edáficas, una propuesta anterior de zonificación de renovales de roble y raulí (Donoso et al. 1993) junto con cartografía proveniente del proyecto "Catastro y Evaluación de Recursos Vegetacionales Nativos de Chile" (CONAF/CONAMA/BIRF 1999).

Diez modelos fustales generales de árbol individual fueron seleccionados considerando que hayan sido previamente usados con éxito en especies de latifoliadas o coníferas y que representen diferentes clases de modelos (cuadro 2). Estimadores de mínimos cuadrados ordinarios fueron utilizados para obtener los parámetros tanto de modelos lineales como no lineales utilizando el software Matlab (MathWorks 2000). Para la comparación de los modelos se calcularon los siguientes estadísticos usando los mismos datos empleados en el ajuste:

$$
\begin{aligned}
& \text { RECM\% }=\frac{100}{\bar{y}}\left[\frac{1}{n} \sum_{i=1}^{n}\left(y_{i}-\hat{y}_{i}\right)^{2}\right]^{1 / 2} \\
& D I F A \%=\frac{100}{\bar{y}}\left[\frac{1}{n} \sum_{i=1}^{n}\left(y_{i}-\hat{y}_{i}\right)\right]
\end{aligned}
$$

donde $y_{i}$ e $\hat{y}_{i}$ corresponden al valor observado y estimado de la medición i-ésima; $\bar{y}$ es el promedio y $n$ el total de observaciones. RECM\% es un estadístico de precisión y DIFA\% de sesgo, y ambos están expresados como una proporción de la media del grupo. Para estos estadísticos, el mejor modelo corresponderá a aquel que presente los valores más cercanos a cero.

Además, se obtuvo un coeficiente de determinación empírico usando la siguiente expresión:

$$
R_{e m p}^{2}=\frac{\sum\left(\hat{y}_{i}-\bar{y}\right)^{2}}{\sum\left(y_{i}-\bar{y}\right)^{2}}=1-\frac{\sum\left(y_{i}-\hat{y}_{i}\right)^{2}}{\sum\left(y_{i}-\bar{y}\right)^{2}}
$$

Producto de que la variable objetivo a estimar pueden ser volúmenes, diámetros o alturas de sección, la selección de los mejores modelos se dividió en etapas. Primero, se 
Cuadro 1. Estadísticos descriptivos para los grupos de ajuste (especie $\times$ zona y general por especie). $n p$ : número total de parcelas, $n a$ : número total de árboles, $n s$ : número total de secciones, $d$ : diámetro a la altura del pecho $(\mathrm{cm}), h$ : altura total $(\mathrm{m})$.

Descriptive statistics for fitting groups (specie $\times$ one and general by specie). $n p$ : number of plots, na: number of trees, $n s$ : number of sections, $d$ : diameter breast height $(\mathrm{cm}), h$ : total height $(\mathrm{m})$.

\begin{tabular}{|c|c|c|c|c|c|c|c|}
\hline Zona & $n p$ & $n a$ & $n s$ & Variable & Mínimo & Media $\pm S$ & Máximo \\
\hline \multicolumn{8}{|c|}{ Raulí } \\
\hline \multirow[t]{2}{*}{1} & 17 & 125 & 1.243 & $d$ & 6,1 & $21,1 \pm 9,0$ & 48,2 \\
\hline & & & & $h$ & 6,6 & $17,8 \pm 5,2$ & 28,1 \\
\hline \multirow[t]{2}{*}{2} & 15 & 56 & 643 & $d$ & 5,9 & $25,6 \pm 9,1$ & 48,7 \\
\hline & & & & $h$ & 8,8 & $20,8 \pm 4,6$ & 27,7 \\
\hline \multirow[t]{2}{*}{4} & 20 & 67 & 770 & $d$ & 9,2 & $23,4 \pm 8,7$ & 49,9 \\
\hline & & & & $h$ & 12,3 & $20,4 \pm 4,4$ & 28,9 \\
\hline \multirow[t]{3}{*}{ Todas } & 53 & 251 & 2.695 & $d$ & 5,9 & $22,9 \pm 9,2$ & 49,9 \\
\hline & & & & $h$ & 6,6 & $19,3 \pm 5,1$ & 31,2 \\
\hline & & & & Roble & & & \\
\hline \multirow[t]{2}{*}{1} & 14 & 41 & 564 & $d$ & 7,8 & $22,9 \pm 11,2$ & 49,1 \\
\hline & & & & $h$ & 10,1 & $20,6 \pm 7,9$ & 35,6 \\
\hline \multirow[t]{2}{*}{2} & 17 & 47 & 625 & $d$ & 7,7 & $24,8 \pm 8,3$ & 45,3 \\
\hline & & & & $h$ & 11,4 & $20,5 \pm 4,9$ & 30,0 \\
\hline \multirow[t]{2}{*}{3} & 9 & 26 & 343 & $d$ & 7,6 & $27,3 \pm 13,4$ & 59,1 \\
\hline & & & & $h$ & 13,3 & $21,6 \pm 5,8$ & 36,8 \\
\hline \multirow[t]{2}{*}{4} & 9 & 66 & 715 & $d$ & 11,5 & $24,3 \pm 7,6$ & 40,1 \\
\hline & & & & $h$ & 11,7 & $21,0 \pm 3,5$ & 27,6 \\
\hline \multirow[t]{3}{*}{ Todas } & 49 & 180 & 2.247 & $d$ & 7,6 & $24,6 \pm 9,7$ & 59,1 \\
\hline & & & & $h$ & 10,1 & $20,8 \pm 5,4$ & 36,8 \\
\hline & & & & Coigüe & & & \\
\hline \multirow[t]{2}{*}{1} & 7 & 20 & 245 & $d$ & 9,5 & $24,5 \pm 11,0$ & 50,2 \\
\hline & & & & $h$ & 11,9 & $19,5 \pm 5,7$ & 30,7 \\
\hline \multirow[t]{2}{*}{4} & 14 & 87 & 966 & $d$ & 6,3 & $24,9 \pm 8,7$ & 44,6 \\
\hline & & & & $h$ & 10,3 & $19,8 \pm 4,1$ & 26,2 \\
\hline \multirow[t]{2}{*}{ Todas } & 28 & 127 & 1.478 & $d$ & 6,3 & $25,3 \pm 9,2$ & 50,2 \\
\hline & & & & $h$ & 10,3 & $19,7 \pm 4,4$ & 30,7 \\
\hline
\end{tabular}

compararon los 10 modelos fustales por grupo de ajuste (especie x zona y general por especie) usando los estadísticos de ajuste basados en la variable respuesta de diámetro de sección sin corteza. De aquí, se preseleccionaron los tres modelos fustales de mejor desempeño para todo el conjunto de ajustes. Esto se realizó utilizando la metodología propuesta por Cao et al. (1980) que consiste en asignar valores consecutivos de acuerdo a los estadísticos al ordenar (jerarquizar) los modelos de mejor a peor, los que son sumados para obtener un índice general.

Posteriormente, a fin de reducir el número de grupos de ajuste se utilizó la prueba parcial F (Rawlings et al. 1998). Con ella se compararon, para cada uno de los modelos preseleccionados, los ajustes de las diferentes zonas de crecimiento de una misma especie con su modelo general (que combina todas las zonas). De este modo se evaluó si es posible combinar todas las zonas de crecimiento de una misma especie en un modelo único.

Finalmente, se analizaron en detalle los tres modelos preseleccionados dividiéndolos en diversas clases de datos y comparándolos en relación a las tres variables objetivo (volumen, diámetro y altura de sección). Esto se realizó en distintas partes del fuste dado que es deseable identificar aquellos modelos de buen desempeño en diferentes partes del fuste o buen ajuste general. Para volumen total individual se definieron las siguientes clases de diámetro a la altura del pecho: $0-10,10-20,20-30$ y $30-60 \mathrm{~cm}$. Los volúmenes reales individuales fueron estimados mediante una interpolación "spline" cúbica (Trincado y Vidal 1999), y los estimados mediante el uso de una integración numérica. 


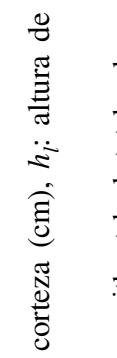

零

:

$\approx$.

$\stackrel{\Xi}{\circlearrowright} \stackrel{\Xi}{\Xi}$

莺

$\ddot{\sigma}$

छิ

웅

ฮั

要 产

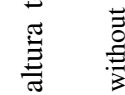

$\ddot{ت} \ddot{\nabla}$

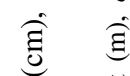

:

व

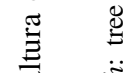

๕

咅

浸

$\ddot{\sim} \dot{\sim} \ddot{0}$

过造

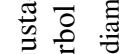

बं बें

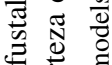

$\exists \cong$

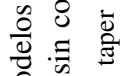

霥

응

卷泀

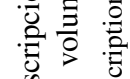

范范

i

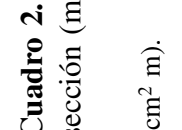

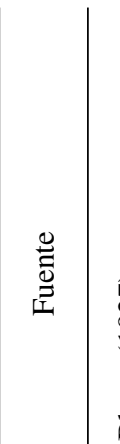

|

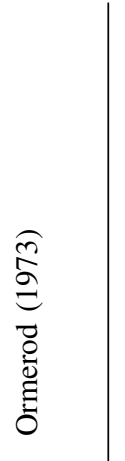

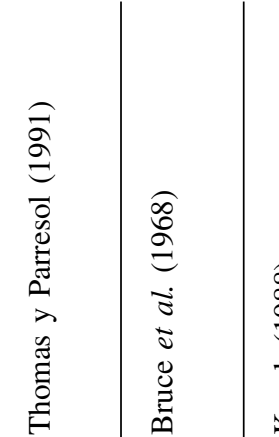

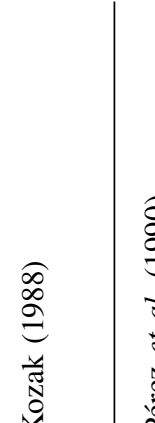

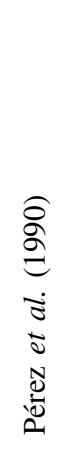

ฮิ

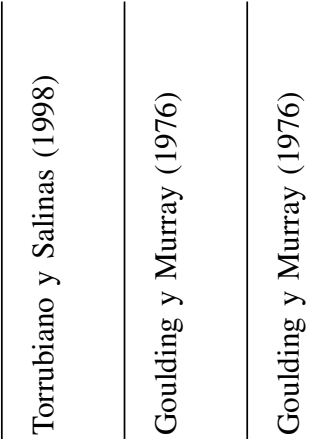

$\stackrel{5}{5}$

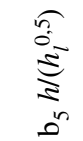

$\overline{8}$
0
0
0

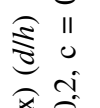

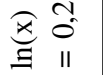

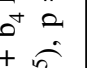

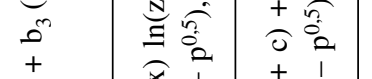

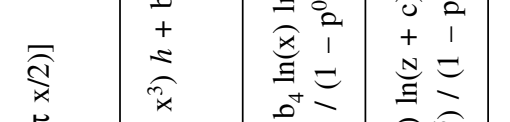

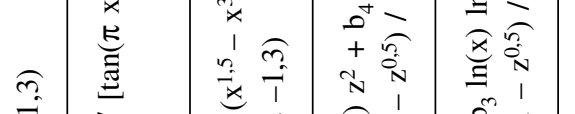

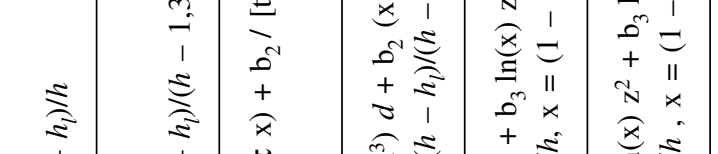

के

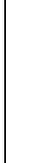

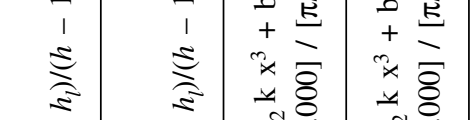

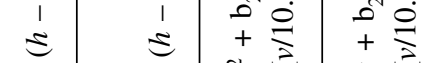

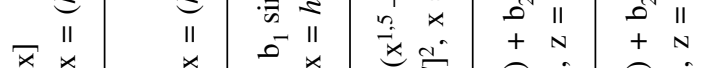

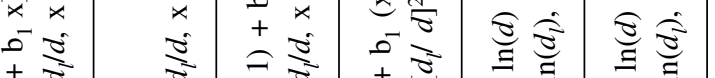

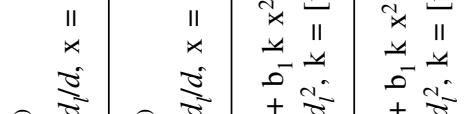

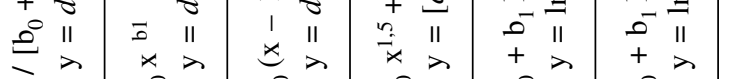

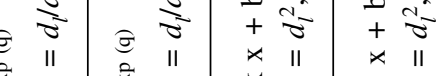

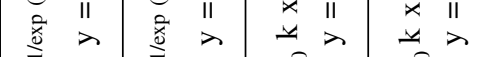

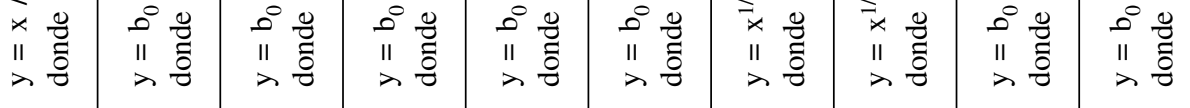

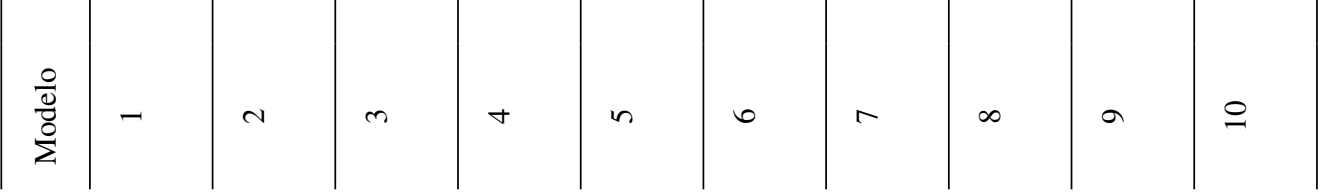


El diámetro $\left(d_{l}\right)$ y la altura de sección $\left(h_{l}\right)$ fueron evaluados usando clases que dividen el perfil en cinco proporciones de la altura total de cada árbol. Los estadísticos RECM\% y DIFA\% fueron calculados para cada clase, modelo y grupo de ajuste, y la selección del modelo final usó la metodología de jerarquización descrita anteriormente. Se consideró una doble ponderación para volumen y diámetro, y simple para altura de sección debido a que esta última usualmente no es primera prioridad.

Adicionalmente, con el objeto de evaluar y comparar la precisión y el sesgo en la estimación de volumen individual de los modelos fustales preseleccionados, para cada uno de los grupos se ajustó la siguiente función de volumen:

$$
v /\left(d^{2} h\right)=b_{0}+b_{1} / d+b_{2} /\left(d^{2} h\right)+b_{3} / h^{2}
$$

donde, $v$ es el volumen total sin corteza del árbol $\left(\mathrm{cm}^{3}\right)$, $d$ es el diámetro a la altura del pecho $(\mathrm{cm})$ y $h$ es la altura total (m). Este modelo fue elegido dado que permite controlar la heterogeneidad de varianzas presente en estimaciones de volumen.

\section{RESULTADOS}

Distintos modelos fustales ocurrieron dentro de los tres mejores para cada grupo de ajuste. Los modelos más frecuentes correspondieron a 4, 5 y 6 y, por otro lado, los modelos 8, 9 y 10 nunca estuvieron dentro de los mejores. Considerando RECM\% y DIFA\% para todos los grupos de ajuste (sin considerar los grupos generales) los menores valores se presentaron para los modelos 4, 5 y 6 , siendo levemente mejores para 5. En relación a las diferentes especies, coigüe presentó los peores estadísticos de ajuste. De este modo, los modelos 4, 5 y 6 fueron preseleccionados para la segunda etapa.

Los resultados de la prueba parcial $\mathrm{F}$ indicaron que únicamente para raulí no existen diferencias entre los ajustes de cada zona de crecimiento con respecto al modelo general. Los valores $P$ de esta prueba obtenidos para raulí fueron de 0,807, 0,996 y 0,999 para los modelos 4, 5 y 6, respectivamente. Por este motivo, se optó por considerar un único grupo de ajuste para raulí. De este modo, se obtuvieron siete grupos de ajuste: uno para raulí, cuatro para roble y dos para coigüe (cuadros 3 y 4).

En relación al diámetro de sección sin corteza, las comparaciones de los modelos 4, 5 y 6 y la función de volumen mostraron valores de RECM\% menores al $10 \%$ para raulí y algo más elevados para roble y coigüe (cuadro 5). Sin embargo, para la mayoría de los grupos de ajuste los resultados de precisión y sesgo fueron aceptables. Los valores de $\mathrm{R}_{\text {emp }}^{2}$ para todos los modelos ajustados son elevados con leves diferencias y valores entre $0,90 \mathrm{y}$ 0,97. En relación al volumen total individual, como es de esperar, los valores de precisión y sesgo fueron peores que para diámetro de sección, con valores de RECM\% entre $10 \%$ y $20 \%$ y sesgos menores al $4 \%$. La excepción fue la especie coigüe para la Zona 1, que presentó valores de RECM\% cercanos al 40\%. Al comparar RECM\% y DIFA\% de los modelos fustales en relación con el modelo de volumen, los valores fueron cercanos e incluso los modelos fustales fueron en algunos casos mejores que el modelo de volumen. Para esta variable, a nivel general, los mejores ajustes se obtuvieron con el modelo 5. La estimación de la altura fustal presentó buenos estadísticos con diferencias mínimas entre los modelos. Sin embargo, al igual que con las otras variables, los valores más altos se encontraron en

Cuadro 3. Parámetros estimados de modelos fustales finales para cada uno de los grupos de ajuste. Expresiones matemáticas se detallan en cuadro 2 .

Estimated parameters for final taper models for each fitted group. Mathematical expressions are detailed in table 2.

\begin{tabular}{|c|c|c|c|c|c|c|c|c|c|}
\hline Zona & Modelo & $\mathrm{b}_{0}$ & $\mathrm{~b}_{1}$ & $\mathrm{~b}_{2}$ & $\mathrm{~b}_{3}$ & $\mathrm{~b}_{4}$ & $b_{5}$ & $b_{6}$ & $b_{7}$ \\
\hline \multicolumn{10}{|c|}{ Raulí } \\
\hline Todas & 5 & 0,04338410 & 0,88657485 & 0,00446052 & 1,97819600 & $-0,40676847$ & 3,50815520 & $-1,84177070$ & 0,19647175 \\
\hline \multicolumn{10}{|c|}{ Roble } \\
\hline 1 & 5 & 0,15921900 & 0,85260316 & 0,00478079 & 1,11507480 & $-0,19946861$ & 1,75732850 & $-0,96843261$ & 0,37186114 \\
\hline 2 & 4 & 0,80469710 & $-0,02339321$ & 0,04233572 & 0,00006908 & 0,01167476 & $-0,00021251$ & - & - \\
\hline 3 & 4 & 0,82191631 & $-0,04593373$ & 0,06184057 & 0,00014938 & 0,01781146 & $-0,00032361$ & - & - \\
\hline 4 & 5 & $-0,20342670$ & 1,03592870 & $-0,00251554$ & 2,00433900 & $-0,42467135$ & 3,81264000 & $-1,99626070$ & 0,23190876 \\
\hline \multicolumn{10}{|c|}{ Coigüe } \\
\hline 1 & 5 & 0,30601932 & 0,78408752 & 0,00741290 & 4,13665070 & $-1,02541230$ & 9,55364480 & $-4,93526510$ & 0,11768003 \\
\hline 4 & 5 & 0,23818537 & 0,82996489 & 0,00510374 & 1,91133230 & $-0,37872755$ & 3,62458410 & $-1,89771910$ & 0,25289679 \\
\hline
\end{tabular}


Cuadro 4. Parámetros estimados del modelo individual de volumen total sin corteza $\left(\mathrm{cm}^{3}\right)$ para cada uno de los grupos de ajuste. Estimated parameters for without bark individual total volume model $\left(\mathrm{cm}^{3}\right)$ for each fitted group.

\begin{tabular}{|c|c|c|c|c|}
\hline Zona & $\mathrm{b}_{0}$ & $\mathrm{~b}_{1}$ & $\mathrm{~b}_{2}$ & $b_{3}$ \\
\hline \multicolumn{5}{|c|}{ Raulí } \\
\hline Todas & 0,2856389 & 0,4143217 & 20,7156010 & $-3,3394867$ \\
\hline \multicolumn{5}{|c|}{ Roble } \\
\hline 1 & 0,2846281 & 1,0349525 & 38,1300780 & $-9,4890117$ \\
\hline 2 & 0,2872324 & 1,4029602 & 11,6150740 & $-18,7095470$ \\
\hline 3 & 0,1759958 & 2,1836258 & $-153,1065900$ & 16,7369030 \\
\hline 4 & 0,3115996 & 0,7751325 & 84,7900110 & $-10,7746180$ \\
\hline \multicolumn{5}{|c|}{ Coigüe } \\
\hline 1 & 0,2833117 & 0,9889675 & 12,3623880 & $-8,5351528$ \\
\hline 4 & 0,2807068 & 1,0938780 & 21,7450290 & $-7,3607826$ \\
\hline
\end{tabular}

Cuadro 5. Estadísticos de precisión (RECM\%) y sesgo (DIFA\%) para las variables volumen total individual sin corteza (v), diámetro de sección sin corteza $\left(d_{l}\right)$ y altura de sección $\left(h_{l}\right)$ para modelos fustales preseleccionados $4,5,6$ y modelo de volumen de árbol individual (Vol). $\mathrm{R}_{\text {emp }}^{2}$, coeficiente de determinación empírico obtenido para variable $d_{l}$.

Error (RECM\%) and bias (DIFA\%) statistics for the variables without bark individual volume $(v)$, without bark section diameter $\left(d_{l}\right)$ and section height $\left(h_{l}\right)$ for pre-selected taper models 4, 5 and 6 together with the individual total volume model (Vol). $\mathrm{R}^{2}{ }_{\text {emp, }}$, empirical determination coefficient obtained for the variable $d_{l}$.

\begin{tabular}{|c|c|c|c|c|c|c|c|c|}
\hline \multirow[b]{2}{*}{ Zona } & \multirow[b]{2}{*}{ Modelo } & \multicolumn{2}{|c|}{$v$} & \multicolumn{2}{|c|}{$d_{l}$} & \multicolumn{2}{|c|}{$h_{l}$} & \multirow{2}{*}{$R_{\mathrm{emp}}^{2}$} \\
\hline & & RECM\% & DIFA\% & RECM\% & DIFA\% & RECM\% & DIFA\% & \\
\hline \multicolumn{9}{|c|}{ Raulí } \\
\hline \multirow{4}{*}{ Todas } & 4 & 14,2 & 0,3 & 9,5 & $-1,0$ & 6,8 & $-0,7$ & 0,964 \\
\hline & 5 & 13,5 & 0,3 & 9,4 & $-0,4$ & 6,9 & $-0,6$ & 0,965 \\
\hline & 6 & 14,4 & 1,4 & 9,5 & $-0,3$ & 6,7 & $-0,4$ & 0,965 \\
\hline & Vol & 13,7 & 0,0 & - & - & - & - & - \\
\hline \multicolumn{9}{|c|}{ Roble } \\
\hline \multirow{4}{*}{1} & 4 & 22,0 & 2,0 & 9,5 & $-0,3$ & 6,7 & $-0,2$ & 0,969 \\
\hline & 5 & 15,8 & 0,1 & 9,6 & $-0,6$ & 6,5 & $-0,3$ & 0,968 \\
\hline & 6 & 19,4 & 1,0 & 9,6 & $-0,5$ & 6,6 & $-0,3$ & 0,968 \\
\hline & Vol & 17,2 & 0,2 & - & - & - & - & - \\
\hline \multirow{4}{*}{2} & 4 & 14,1 & $-1,5$ & 10,1 & $-0,2$ & 7,6 & $-0,1$ & 0,948 \\
\hline & 5 & 22,1 & $-4,6$ & 11,1 & $-0,4$ & 7,6 & 0,1 & 0,937 \\
\hline & 6 & 16,8 & $-2,9$ & 10,7 & $-0,3$ & 7,7 & 0,5 & 0,942 \\
\hline & Vol & 15,4 & $-0,5$ & - & - & - & - & - \\
\hline \multirow{4}{*}{3} & 4 & 16,3 & $-3,4$ & 11,6 & $-0,7$ & 7,0 & $-0,6$ & 0,952 \\
\hline & 5 & 16,4 & 3,9 & 11,2 & 0,0 & 7,5 & $-0,8$ & 0,955 \\
\hline & 6 & 13,3 & $-0,7$ & 11,9 & $-0,2$ & 7,7 & $-0,1$ & 0,950 \\
\hline & Vol & 12,7 & $-2,2$ & - & - & - & - & - \\
\hline \multirow{4}{*}{4} & 4 & 11,0 & 0,5 & 8,0 & $-0,4$ & 6,1 & $-0,3$ & 0,963 \\
\hline & 5 & 11,1 & 0,6 & 8,0 & $-0,24$ & 6,0 & $-0,2$ & 0,963 \\
\hline & 6 & 10,9 & 0,6 & 8,1 & $-0,24$ & 5,9 & 0,0 & 0,962 \\
\hline & Vol & 10,4 & 0,0 & - & - & - & - & - \\
\hline \multicolumn{9}{|c|}{ Coigüe } \\
\hline \multirow{3}{*}{1} & 4 & 41,3 & 3,0 & 16,1 & $-0,2$ & 9,3 & $-0,1$ & 0,903 \\
\hline & 5 & 33,7 & $-0,4$ & 15,2 & 0,4 & 10,1 & 0,8 & 0,913 \\
\hline & 6 & 38,3 & 8,3 & 15,6 & 1,0 & 11,2 & 2,3 & 0,909 \\
\hline \multirow{5}{*}{4} & Vol & 35,7 & 0,0 & - & - & - & - & - \\
\hline & 4 & 16,3 & 1,2 & 9,2 & $-1,3$ & 6,5 & $-0,8$ & 0,962 \\
\hline & 5 & 15,6 & 1,7 & 8,7 & $-0,3$ & 6,3 & $-0,3$ & 0,966 \\
\hline & 6 & 16,7 & 2,8 & 8,8 & $-0,2$ & 6,2 & $-0,1$ & 0,965 \\
\hline & Vol & 15,7 & 0,7 & - & - & - & - & - \\
\hline
\end{tabular}


coigüe para la Zona 1. Resultados similares se presentaron para el $R_{\text {emp }}^{2}$ pero en todos los casos sus valores fueron mayores a 0,90 .

Al implementar la jerarquización de modelos por clases para las variables $v, d_{l}$ y $h_{l}$, los modelos 4 y 5 resultaron ser los mejores. Sin embargo, las diferencias entre estos dos modelos fueron mínimas. A modo de ejemplo, los valores de los estadísticos para raulí se muestran en la figura 1 para las variables $v$ y $d_{l}$. En ella se observa que no existieron mayores diferencias entre los modelos. Sin embargo, para volumen en los modelos 4 y 6 se encontró una sobrestimación en los árboles más pequeños (clase $0-10 \mathrm{~cm} \mathrm{de} d$ ). Para $d_{l}$ se observó un incremento notorio en RECM\% a medida que se consideraron alturas mayores en el perfil, llegando a ser superiores al $20 \%$. Pero, en la práctica, éste tiene un efecto mínimo, dado que la mayoría del volumen se concentra en los primeros dos tercios del árbol. Destaca que para el modelo 4 se obtuvieron valores de DIFA\% cercanos al $-15 \%$. Situaciones similares se detectaron en los otros grupos de ajustes.

\section{DISCUSIÓN}

La mayoría de los modelos seleccionados presentaron valores de precisión menores al $15 \%$ en la estimación del diámetro de sección sin corteza. La única excepción fue para coigüe en la Zona 1, lo que se puede deber al reducido número de observaciones (sólo 20 árboles). La precisión y sesgo de las predicciones de volumen utilizando modelos fustales son de similar calidad al modelo de volumen individual evaluado. En general, no se encontraron grandes diferencias entre los modelos preseleccionados, en donde los mejores ajustes fueron obtenidos con los modelos de Bruce et al. (1968) y Kozak (1988) con resultados levemente mejores para este último. En la literatura existe un diverso grupo de modelos de ahusamiento disponible. En un estudio de compatibilidad de funciones de volumen y ahusamiento para renovales de roble en la región de los Lagos (Pérez 2004), en que se probaron dos modelos de volumen y ahusamiento para tres zonas de crecimiento, se obtuvo que los modelos estimadores de volumen presentan sesgos (DIFA\%) negativos, lo que indica sobrestimación del volumen total. En cuanto a error de estimación (RECM\%) ambos modelos presentan valores superiores al 14\%, lo cual es considerado alto, ya que normalmente se espera tener errores de entre un 5\% y $10 \%$. Por lo tanto, los modelos de volumen total en ambas zonas de crecimiento no cumplieron con los requisitos de error y sesgo permitidos, entregando estimaciones de volumen total deficientes. Por otra parte, Higuera (1994) realizó el ajuste y validación de seis funciones de volumen para roble, siendo el modelo de Näslund el más destacado con un sesgo que varió entre $-5,7 \%$ y $2,3 \%$ y un error entre $6,4 \%$ y $14 \%$.

Como se aprecia en la figura 1 , tanto el error como el sesgo presentan valores relativamente bajos hasta el diámetro 40-60 cm. La variabilidad en árboles de mayor diámetro se manifiesta en la tendencia de aumento de estos dos estadísticos. Sería recomendable realizar los ajustes para volumen, mediante funciones de volumen o ahusamiento, en dos o tres estratos de diámetro.

Otro estudio desarrollado por Hueitra (2004), en donde se probaron siete funciones de volumen y once de ahusamiento (Higuera 1994) para renovales de roble de las Regiones de
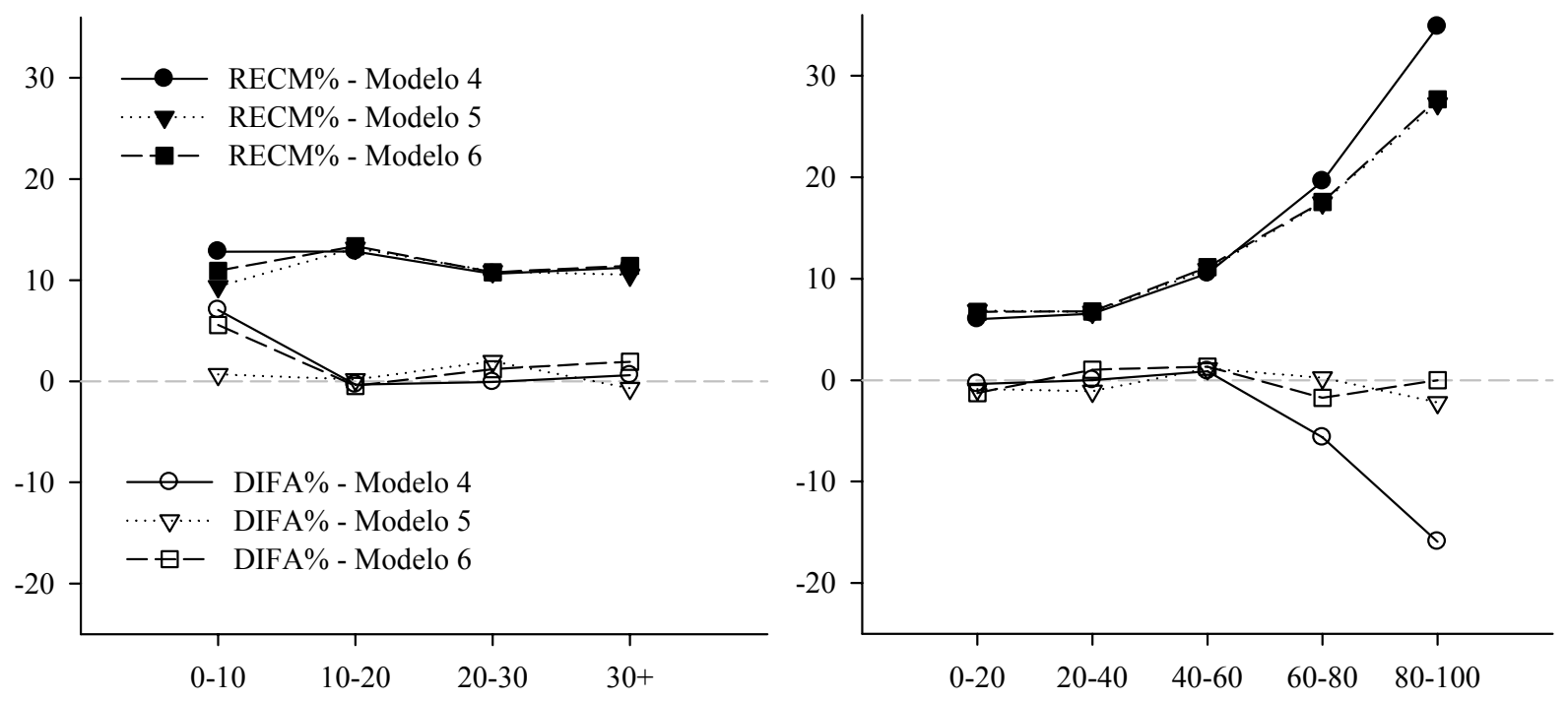

Figura 1. Estadísticos de ajuste por clase de diámetro para raulí en todas las zonas para las estimaciones de volumen total sin corteza del árbol (izquierda) y diámetro sección sin corteza (derecha) para los modelos de ahusamiento 4, 5 y 6 (cuadro 2). Eje X: clases de diámetro a la altura del pecho $(\mathrm{cm})$.

Raulí's fitting statistics in all growth zones for without bark individual total volume estimations (left) and without bark section diameter (right) on taper models 4, 5 and 6 (table 2). X-axis contains diameter at breast height classes (cm). 
La Araucanía y de Los Lagos, arrojó que para los modelos de volumen existe un error con tendencia a la subestimación que no sobrepasa el 10\%. Para los modelos fustales los errores a lo largo del fuste son menores al $10 \%$ y con tendencia a la sobrestimación y se encontró que las funciones de volumen predicen con mayor exactitud este parámetro que las funciones transformadas de ahusamiento.

Pese a lo anterior, en los trabajos de ajuste de funciones de volumen y ahusamiento para Nothofagus spp. de Higuera (1994) y de Vidal (1998) se indican las ventajas que puede tener el ajuste de funciones de ahusamiento para establecer predictores de volumen. Esto se refiere a que una vez integradas pueden entregar un valor de volumen más preciso. Otra ventaja es que como se puede elegir el índice de utilización, la función puede ser útil ante los cambios y exigencias externas como los mercados.

Respecto a la estimación de alturas a distintos diámetros a lo largo del fuste, Pérez (2004) señala que la utilización de los modelos de ahusamiento probados en dicho estudio son poco recomendables, ya que cumplen con requisitos de error y sesgo pero sólo en puntos aislados en el fuste y no en forma continua, lo cual no permite abarcar secciones más extensas. En cuanto a estimación de diámetros a distintas alturas a lo largo del fuste, el mismo autor señala que los modelos probados poseen distintos valores de sesgo, por lo que tienden a sobrestimar y subestimar para las distintas zonas de crecimiento; y para el error señala que a mayor altura (70\% del fuste) menor es el porcentaje de error $(<10 \%)$. Finalmente se señala que al analizar el sesgo y error en forma conjunta sólo en algunas secciones del fuste se cumplen los requisitos permitidos, haciendo difícil su utilización continua a lo largo del fuste, y que se podría utilizar ya sea para la parte basal, central o superior del árbol.

Por lo tanto, es extremadamente difícil obtener una función que describa adecuadamente todas las porciones del fuste. Para la estimación de volumen individual, la mejor opción es, sin lugar a dudas, el ajuste de un modelo de volumen. Sin embargo, la ventaja de usar modelos de ahusamiento, como los presentados en este estudio, es que éstos permiten estimar volumen de trozas de cualquier dimensión. En este trabajo se encontró que las pérdidas en precisión y sesgo para la estimación del volumen individual total fueron mínimas, justificando el uso de estos modelos.

Los resultados presentados permiten el uso de estos modelos en condiciones similares a las del origen de los datos. Es decir, en renovales jóvenes con niveles nulos o bajos de alteración antrópica, con diámetros no superiores a $50 \mathrm{~cm}$ (raulí y coigüe) o $60 \mathrm{~cm}$ (roble) y que se encuentren entre los $36^{\circ}$ y $42^{\circ} \mathrm{S}$ en Chile. Sin embargo, al ser obtenidos de una base de gran cobertura geográfica, se espera que estos modelos sean de utilidad para un grupo amplio de condiciones. De todos modos, se recomienda la calibración y evaluación de esta información con un muestreo complementario de tamaño reducido.
En resumen, se recomienda el uso de los parámetros estimados para los grupos de ajuste. Sin embargo, se recomienda usar con cautela el modelo obtenido para coigüe en la Zona 1.

\section{AGRADECIMIENTOS}

La mayor parte de este estudio fue desarrollado durante la ejecución del proyecto FONDEF D97I1065 "Software de Planificación de Actividades en Renovales de Roble, Raulí y Coigüe en la IX y X Región" de la Universidad Austral de Chile, el que fue financiado por CONICYT junto a empresas e instituciones asociadas. Se agradece el aporte de valiosa información adicional de estudios realizados por el Dr. Hans Grosse (Instituto Forestal) y el Dr. Pablo Donoso (Universidad Austral de Chile), además del trabajo de varios brigadistas de terreno.

\section{REFERENCIAS}

Bruce D, R Curtis, C Vancoevering. 1968. Development of a system of taper and volume tables for red alder. Forest Science 14(3): 339-350.

Bhere CE. 1927. Form class taper tables and volume tables and their application. J. Agric. Res. 35: 673-744.

Cao Q, H Burkhart, T Max. 1980. Evaluation of two methods for cubic-volume prediction of loblolly pine to any merchantable limit. Forest Science 26(1): 71-80.

CONAF (Corporación Nacional Forestal, CL), CONAMA (Comisión Nacional del Medio Ambiente, CL), BIRF (Banco Internacional de Reconstrucción y Fomento, USA), Universidad Austral de Chile, Pontificia Universidad Católica de Chile, Universidad Católica de Temuco. 1999. Proyecto catastro y evaluación de los recursos vegetacionales nativos de Chile. Santiago, Chile. CONAF. 87 p.

Donoso P, C Donoso, V Sandoval. 1993. Proposición de zonas de crecimiento de renovales de roble (Nothofagus obliqua) y raulí (Nothofagus alpina) en su rango de distribución natural. Bosque 14(2): 37-55.

Goulding C, J Murray. 1976. Polynomial taper equations that are compatible with tree volume equations. New Zealand Journal of Forest Science 5(3): 313-322.

Grosse H, V Cubillos. 1991. Antecedentes generales para el manejo de renovales de raulí, roble, coigüe y tepa. Informe Técnico $\mathrm{N}^{\circ}$ 127. Santiago, Chile. INFOR-CORFO. 47 p.

Higuera C. 1994. Funciones de volumen y ahusamiento para roble (Nothofagus obliqua) y hualo (Nothofagus alessandrii). Tesis Ingeniero Forestal. Valdivia, Chile. Facultad de Ciencias Forestales, Universidad Austral de Chile. 74 p.

Hueitra R. 2004. Funciones de volumen y ahusamiento para renovales de roble (Nothofagus obliqua [Mirb.] Oerst.) en zonas de crecimiento, en la IX y X Región. Tesis Ingeniero Forestal. Valdivia, Chile. Facultad de Ciencias Forestales, Universidad Austral de Chile. 47 p.

Kahler C. 1993. Determinación de una función de ahusamiento para renovales de roble y raulí. Ciencia e Investigación Forestal 7(1): 117-133. 
Kozak A. 1988. A variable-exponent taper equation. Canadian Journal of Forest Research 18(11): 1363-1368.

MathWorks. 2000. Using MATLAB Version 6.0. Natick, MA, USA. The MathWorks Inc. 874 p.

Max TA, HE Burkhart. 1976. Segmented-polynomial regression applied to taper equations. Forest Science 22(3): 283-289.

Newnham RM. 1992. Variable-form taper functions for four Alberta tree species. Canadian Journal of Forest Research 22(2): 210-223.

Ormerod DW. 1973. A simple bole model. For. Chron. 49: 136-138.

Ortega A, S Gezan. 1998. Cuantificación de crecimiento y proyección de calidad en Nothofagus. Bosque 19(1): 123-126.

Pérez M. 2004. Compatibilidad de funciones de volumen y ahusamiento para renovales de roble en la Décima Región. Tesis
Ingeniero Forestal. Valdivia, Chile. Facultad de Ciencias Forestales, Universidad Austral de Chile. 32 p.

Prodan M, R Peters, F Cox, P Real. 1997. Mensura Forestal. San José, Costa Rica. IICA-GTZ. 561 p.

Rawlings JO, SG Pantula, DA Dickey. 1998. Applied Regression Analysis: A Research Tool. New York, USA. SpringerVerlag. $657 \mathrm{p}$.

Torrubiano C, H Salinas. 2008. Herramientas de cubicación para pino oregón (Pseudotsuga menziesii (Mirb) Franco) ubicado en la zona de Valdivia. Bosque 19(2): 11-21.

Thomas CE, BR Parresol. 1991. Simple, flexible, trigonometric taper equations. Canadian Journal of Forest Research 21(7): 1132-1137.

Trincado G, J Vidal. 1999. Aplicación de interpolación "spline" cúbica en la estimación de volumen. Bosque 20(2): 3-8.

Recibido: 18.06 .07

Aceptado: 12.03.09 